\title{
"Warranted assertibility" im wissenschaftlichen und politischen Diskurs am Beispiel der Diskussionen um die Bildungsstandards
}

\section{Jean-Luc Patry}

Der Aufsatz von Walter Herzog, "Unterwegs zur 08/15-Schule? Wider die Instrumentalisierung der Erziehungswissenschaft durch die Bildungspolitik», wird kritisch diskutiert. Inhaltlich wird Herzog in vielen Fragen Recht gegeben. Allerdings wird kritisiert, dass Herzog insbesondere wegen deren Verwendung bestimmter Begriffe den Politikern unangemessenes Vorgehen unterstellt. Die Botschaften von Herzog sind nicht grundsätzlich falsch, aber die Argumentation ist unangemessen und polemisch. In einzelnen Bereichen werden weitere Argumente angedeutet. Herzogs Polemik ist zu bedauern, da wichtige Botschaften nicht argumentativ gestützt werden und deshalb durch die Adressaten leicht als unwissenschaftlich abgetan werden können.

Thema des Aufsatzes von Walter Herzog, «Unterwegs zur 08/15-Schule? Wider die Instrumentalisierung der Erziehungswissenschaft durch die Bildungspolitik» ist die Rolle der Bildungsforschung in der Schul- und Bildungspolitik in der Schweiz am Beispiel der laufenden Reformen im obligatorischen Schulbereich in der Schweiz und insbesondere im Hinblick auf die nationalen Bildungsstandards. Ich möchte in meiner Reaktion weder auf die spezifischen wissenschaftlichen Grundlagen noch auf die spezifischen politischen Rahmenbedingungen in der Schweiz eingehen, weil ich deren Entwicklungen nicht im Detail verfolgen konnte, sondern möchte mich auf die (mögliche) Rolle der Bildungsforschung in der Politik konzentrieren und den Aufsatz von Herzog zum Anlass nehmen, einige mir wichtige Aspekte anzusprechen; dies wird dann die Möglichkeit bieten, spezifisch auf das Thema der Standards einzugehen.

Instrumentelle Rolle der Erziehungswissenschaft und $\mathrm{Bi}$ ldungs forschung

Selbstverständlich sollen Erziehungswissenschaft und Bildungsforschung der Politik helfen, angemessene Entscheidungen zu treffen. Erziehungswissenschaft ist 
ja nicht nur eine Wissenschaft von der Praxis, sondern auch für die Praxis. Aber ebenso selbstverständlich sollen die entsprechenden Wissenschaftler die Grundprinzipien ihrer Wissenschaft deswegen nicht aufgeben. Auf der anderen Seite sind Wissenschaftler immer und zwangsläufig in Dilemmata verschiedener Art: in ethischer, methodischer, sozialer, rechtlicher, ökonomischer, gesellschaftlicher und persönlicher Hinsicht (Gastager \& Patry, 2006). Wissenschaftler werden also nie irgendwelche Maximumforderungen erfüllen können, sondern immer Kompromisse schließen müssen. Dies gilt insbesondere auch für jene Wissenschaftsbereiche, die sich mit gesellschaftlich relevanten Fragen befassen. Die gesellschaftlichen Werte spielen bei allen Forschungsaktivitäten eine Rolle (Patry \& Patry, 2003), sind aber bei den letztgenannten Wissenschaftsbereichen von besonderer Bedeutung, und gegebenenfalls müssen in anderen Bereichen Abstriche gemacht werden. Ein klassisches Beispiel ist die Evaluationsforschung. So hat House (z.B. 1993; House \& Howe, 2000, S. 3-12) immer wieder deutlich gemacht, dass Evaluation letztlich der human welfare zu dienen hat und andere Ziele dem unterzuordnen sind.

Allerdings darf dies m.E. nicht so weit gehen, dass wissenschaftliche Ansprüche, insbesondere die Rationalität und intellektuelle Redlichkeit, aufgegeben werden. Hier ist Herzog zuzustimmen, und ebenso, dass nicht Evidenzen, sondern Argumente den Kern der wissenschaftlichen Rationalität bilden; es ist auch angemessener, mit Dewey (zit. nach Phillips \& Burbules, 2000, S. 31) von «warranted assertibility" (was ich mit "gerechtfertigte Behauptbarkeit» übersetzen möchte) statt von Wahrheit zu sprechen. "Warranted assertibility» aber beruht immer und notwendiger Weise auf Theorien (schon Hanson, 1958) - auch hier stimme ich mit Herzog überein.

Zur Rationalität und intellektuellen Redlichkeit gehört auch, dass sich Wissenschaftler in ihrer Tätigkeit nicht der Werturteile enthalten können: Wie jede Tätigkeit ist auch wissenschaftliches Tun wertgeladen, und angesichts der gesellschaftlichen Bedeutung der Wissenschaft haben Wissenschaftler eine besondere Verantwortung. Zur intellektuellen Redlichkeit gehört dann aber auch, diese Werthaltungen, so weit sie den Forschungsprozess und dessen - insbesondere gesellschaftlichen - Auswirkungen betreffen, transparent zu machen; aus diesem Grunde ist eine wertfreie Wissenschaft auch als System von Sätzen letztlich nicht haltbar (vgl. auch Zecha, 1984; s. Patry, 2006 für Details). In Wissenschaft (als System von Sätzen), die unmittelbar gesellschaftlichen Zwecken dient, gilt dies in besonderem Maße.

Allerdings muss man sich bewusst sein, dass diese Prinzipien in der Öffentlichkeit wenig bekannt und schon gar nicht diskutiert werden. Selbst viele Wissenschaftler haben sich diesbezüglich noch kaum Gedanken gemacht. Die Aussage von Herzog, "Wenn es wissenschaftliche Erkenntnis ohne theoretische Annahmen nicht gibt, dann kann der Versuch der Indienstnahme der Forschung durch die Politik nur bedeuten, dass von der Theorielosigkeit der Reform des schweizerischen Bildungssystems abgelenkt werden soll», ist auf diesem Hinter- 
grund problematisch. Das Ziel der Ablenkung von der Theorielosigkeit der Reform ist eine Unterstellung, die durch Herzog nicht hinreichend argumentativ unterstützt ("warranted») wird. Es ist nicht auszuschliessen, dass «die Politik» im besten Glauben wissenschaftliche Hilfe in Anspruch nahm. Inwiefern diese dann auch theorielos war, kann ich nicht beurteilen.

Die Wissenschaftler sind dann aber jedenfalls in die Pflicht zu nehmen, die wissenschaftlichen Prinzipien in ihren Verhandlungen mit politischen Instanzen einzumahnen. Ob sie dies im vorliegenden Fall auch getan haben, kann ich nicht beurteilen. Die Erfahrung in anderen Kontexten sind unterschiedlich: Es gibt Wissenschaftler, die dies explizit tun (genannt sei etwa House, dessen Einsatz für human welfare oben erwähnt wurde), andere halten sich diesbezüglich zurück, sei es aus Ignoranz, sei es, dass sie es nicht für wichtig oder relevant erachten, sei es aus anderen Gründen. Auf der anderen Seite sind die Politiker in die Pflicht zu nehmen, die genannten Prinzipien anzuerkennen, wenn sie - was sinnvoll ist - wissenschaftliche Dienste in Anspruch nehmen. Wenn Herzogs Beitrag diese Funktion hat, kann dem nur zugestimmt werden, und es ist wünschbar, dass diesbezüglich Fortschritte erzielt werden, nicht nur im Bereich der Bildungsstandards, sondern im Bereich der wissenschaftlichen Politikberatung im Allgemeinen.

\section{Ziele und Standards}

Herzog verweist hinsichtlich des Begriffs «Standard» auf dessen Herkunft aus der industriellen Fertigung und Normierung. Gleiche Wörter haben aber nicht notwendiger Weise die gleiche Bedeutung. «Standards» in der Bildung bedeutet dementsprechend nicht unbedingt Normierung im Sinne einer Maschinengewehrherstellung.

Bei den Standards geht es letztlich um Ziele; nicht um Normen für Gegenstände. In der Fertigungstechnik gibt es nur äusserst geringe Toleranzen, sowohl nach oben wie nach unten («einheitliche Präzision»). Eine solche Konzeption wäre im Erziehungsbereich zweifellos unangemessen: Eine solche Punktgenauigkeit ist selbstverständlich nicht erreichbar und auch nicht sinnvoll; wenn die Standards übertroffen werden, ist dies jedenfalls zu begrüssen. Es ist erneut sehr fragwürdig, zu unterstellen, "die Politik» habe das Anliegen der einheitlichen Präzision.

Die Polemik ist gar nicht notwendig. Angemessener als der Verweis auf Fertigungstechnik mit dem Verweis auf «08/15» und den sicherlich beabsichtigten entsprechenden Konnotationen - die dem Reformprojekt aber nicht gerecht werden - wäre wohl eine Bezugnahme auf Magers (1977) Lehrzieloperationalisierungen: Wie bei den Bildungsstandards geht es auch dort um Messungen zur Operationalisierung der Ziele mit dem Effekt, dass dann nur Ziel sein kann, was gemessen werden kann. Dies zu kritisieren ist ja eines der Ziele von Herzog (vgl. dazu unten, Abschnitt 4). 


\section{Input und output}

Ganz analog wie im zweiten Kapitel argumentiert Herzog, wenn er zur Untermauerung der unterstellten Absicht der Politiker die Analogie zur industriellen Fertigung verwendet mit der Behauptung, dort gelte die Outputsteuerung, die auch im Bildungsbereich eingeführt werden solle, während dort bislang die Inputsteuerung durch Verordnungen etc. dominiert habe. Aber die letztgenannte Vorstellung steht im Widerspruch zum Konzept von Erziehung: Erziehung und Bildung sind immer zielorientiert, dies gilt auch für die Schule, in der ja Lehrpläne nicht nur Input, sondern auch Output betreffen. Dies kommt vor allem in den Vorwörtern der Lehrpläne zum Ausdruck, wo wenn auch in recht vagen Formulierungen Erziehungsziele formuliert werden. Auch die konkreten Kapitel der Curricula dienen nicht nur der Steuerung des Unterrichts, sondern thematisieren Ziele, wie überhaupt so genannte Input-Steuerungs-Elemente letztlich dem Erreichen von Zielen dienen und deshalb die Trennung von Input und Output in diesem Zusammenhang nicht ganz durchgezogen werden kann.

Dass die Vorgaben wie das Prinzip der Jahrgangsklassen, die Übertrittsverfahren und was der Vorschriften mehr sind, Selbstzweck seien, wird wohl niemand behaupten. Vielmehr dienen sie letztlich wiederum dem Ziel der Bildung. Auch dass sie diesbezüglich optimal seien, kann allerdings bezweifelt werden, und deshalb ist eine Reform notwendig.

Herzog kritisiert die Orientierung (um den von ihm zu Recht kritisierten Begriff «Steuerung» nicht zu verwenden) am Output statt am Input nicht ausdrück\$lich, der Vergleich mit Fertigungsprozessen legt dies aber nahe. Gerechtfertigt ist eine solche Kritik jedenfalls nicht, da Erziehungs- und Bildungserfolge sich letztlich darin erweisen, was Erziehung bewirkt, nicht ob sie vorschriftsgemäss erfolgte. $\mathrm{Ob}$ es dazu nationaler Standards bedarf, ist aber eine andere Frage.

Es ist nicht nachvollziehbar, warum die Einführung nationaler Bildungsstandards ein Nationendenken bewirken sollte, wie es PISA tut. PISA bewirkte de facto ein solches Denken, ob beabsichtigt oder nicht, weil Vergleiche zwischen Ländern realisiert werden. Dass auch eine kriterielle Bezugsnorm verwendet wird, ist der Öffentlichkeit weitgehend entgangen; dies wurde von den PISA Verantwortlichen auch zu wenig publik gemacht. Aber ein Ländervergleich wie in PISA ist ja mit Bildungsstandards nicht beabsichtigt: Es ist nicht anzunehmen, dass in der Öffentlichkeit - und darum geht es beim Nationendenken ja letztlich - Standards verschiedener Länder gegeneinander ausgespielt werden, wie es mit PISA-Ergebnissen getan wird. Die Kritik an den Standards, diese seien national und förderten deshalb den Nationalismus, ist also nicht gerechtfertigt. Wenn dieser Punkt zu kritisieren ist, dann mit anderen Gründen (vgl. unten). 


\section{Thorndike and beyond}

Das vierte Kapitel beginnt erneut mit einer Unterstellung: «dass Bildungsstandards weniger Ausdruck eines pädagogischen als eines politischen Interesses» seien (Auszeichnung im Original). Diese Behauptung ist nicht gerechtfertigt, weil sie auf der problematischen Argumentation des vorhergehenden Kapitels aufbaut. Hinzu kommt aber, dass Herzog offenbar implizite «politische Interessen» mit "versuchter Einflussnahme» gleichsetzt und dies negativ wertet. Das reine Faktum einer Einflussnahme steht aber nicht notwendiger Weise im Widerspruch zu pädagogischen Zielen, auch der Versuch der Einflussnahme im Hinblick auf eine Vereinheitlichung der Strukturen, Ziele, Inhalte und Methoden ist nicht per se unpädagogisch, wenn diese pädagogisch legitimiert sind. Die Frage ist also zu stellen, ob diese Legitimierung im vorliegenden Falle gegeben ist. Wenn nicht, ist auf das erste Kapitel zu verweisen: auf die Notwendigkeit einer angemessenen Theorie.

Das Kapitel «Thorndike lässt grüssen» verweist auf zwei weitere Aspekte: einerseits auf die Steuerung und andererseits auf die Messbarkeit. Betrachten wir zunächst die Steuerung. Es ist Herzog zuzustimmen, dass Steuerung hier unangemessen ist (sein Abschnitt 5.1, «Drittens»). «Regelung» ist hier m.E. der bessere Begriff, nun aber nicht als automatisches Korrigieren von Abweichungen von Sollwerten, wie Herzog unterstellt, sondern dahingehend, dass der Akteur handelt (etwas tut) und sich Feedback holt, ob und wie weit er darin erfolgreich gewesen ist, und sein Tun entsprechend korrigiert. Dies ist schon im TOTE-Modell von Miller, Galanter und Pribram $(1960)^{1}$ und seither immer wieder thematisiert worden.

Es ist richtig, dass diese Regelung je nach Systemebene - Interaktion des Lehrers mit dem Schüler, Organisation einer Schule, Gestaltung eines Schulsystems - unterschiedliche Grundlagen braucht; wir haben dies in einem anderen Kontext (Lehrerbildung; vgl. Patry et al., 2006, S. 9-27) differenziert analysiert. Die Verwendung von Messungen, die zum Zwecke der Systemregelung entwickelt wurden, für die Selektion ist grundsätzlich problematisch. Dabei sind es nicht die Erfahrungen, auf die Herzog verweist, die letztlich entscheidend sind: Dass die schwachen Schülerinnen und Schüler noch schwächer werden, die Lehrerzentriertheit zunimmt etc., sind nicht automatische Konsequenzen von «teaching to the test», sondern die Folge von falschen Tests; dies verweist also auf das, was gemessen wird, und dies wird unten noch zu diskutieren sein. Das Problem ist vielmehr, dass die entsprechenden Instrumente unterschiedlich zu konstruieren sind. Für Selektionszwecke ist es jedenfalls unangemessen, alle Schülerinnen und Schüler über einen Leisten zu schlagen (im Sinne nationaler Standards), sondern die individuellen Gegebenheiten unter Einschluss der Vorhersage der weiteren Entwicklung des Individuums sind zu berücksichtigen, so dass das Kriterium von Person zu Person unterschiedlich sein wird. 
Das ist aber nicht der Hauptkritikpunkt an den Standards. Das Entscheidende ist, welches Kriterium für den «Test» im Sinne des TOTE-Modells verwendet wird. Kriterium kann nach der von Herzog vermuteten «Philosophie» bei der Formulierung der Bildungsstandards nur sein, was auch messbar ist. An sich ist die Argumentation verlockend, mittels kunstgerechter Messungen festzustellen, ob die Ziele erreicht worden sind oder nicht. Problematisch wird es dann, wenn nur mehr als Ziel formuliert werden kann, was auch gemessen werden kann. Dies führt zu einer Begrenzung der Zielvielfalt, die nicht normativ begründet ist, wie es auf Grund der non-naturalistischen Metaethik, die hier vertreten wird, zu fordern ist, sondern auf Grund von Überlegungen, die nichts mit der Zielwahl selber zu tun haben. Eine solche Begründung ist nicht gerechtfertigt.

Ziele von Erziehung und Bildung sind nicht nur Kompetenzen (und hier soll auch das Schulwissen als Kompetenz interpretiert werden). Unter «Kompetenz» wird hier eine Fähigkeit, d.h. das, was eine Person zu tun in der Lage ist, wenn die Bedingungen optimal sind. Im Sinne von Cronbach (1970, S. 35) handelt es sich um «maximale Performanz»: In den entsprechenden Erhebungssituationen bemüht sich der Protagonist, sein Bestes zu geben, an die Grenzen seiner Möglichkeiten zu gehen. Das ist charakteristisch für die Schule mit ihren Prüfungen, wo die Schülerinnen und Schüler mit wenigen Ausnahmen bemüht sind, möglichst gute Leistungen zu zeigen. Das ist auch so für Tests wie PISA, und das gilt auch für Standards.

Im Alltag, für den die Schülerinnen und Schüler letztlich lernen, spielen maximale Performanzen aber eine kleine Rolle. Wir sind selten damit konfrontiert, an die Grenzen unserer Möglichkeiten zu gehen. Gerade in Bereichen wie Sozialverhalten, Verantwortung etc. praktizieren wir, was Cronbach (ebenda) die «typische Performanz» genannt hat: Typische Performanz bezieht sich darauf, was jemand wahrscheinlich in einer bestimmten Situation tun wird. Hier geht es nicht darum, ein einzelnes Ziel - möglichst gute Leistung - zu realisieren, sondern es werden meistens mehrere Ziele gleichzeitig angestrebt, was dazu führt, dass erstens Kompromisse zwischen den Zielen einzugehen sind (Patry, 1996) und zweitens die optimale Handlung von Situation zu Situation unterschiedlich ist (Patry, 1991).

Während die maximale Performanz relativ einfach valide zu messen ist - man muss die oben erwähnte angemessene Erhebungssituation herstellen, valide Aufgaben geben und die Akteure auffordern, ihr Bestes zu geben. Demgegenüber sind Dispositionen, die zur typischen Performanz gehören, nur sehr schwer zu messen. Man kann sie zwar in einer gewissen Situation erfassen, aber sie sind äusserst anfällig auf biases. Man kann den Akteuren nicht sagen, sie sollten sich möglichst kompetent verhalten, sondern man bittet sie allenfalls, möglichst ehrlich zu sein (wenn es sich um einen Fragebogen handelt), oder man beobachtet sie ohne ihr Wissen oder täuscht sie - alles Vorgehensweisen, die bei maximaler Performanz nicht notwendig sind. Dass viele dieser Verfahren auch unethisch sind, sei hier nur am Rande erwähnt; im vorliegenden Zusammenhang viel 
wichtiger ist, dass sie nicht valide sind (Mischel, 1968) - aus theoretischen Gründen nicht valide sein können.

Entsprechend gibt es für die Leistungsbeurteilung wie für die Bildungsstandards Wissenstests, Kompetenzerhebungen etc., also Erhebungen der maximalen Performanz, aber keine Messungen zum Sozialverhalten, zu den Einstellungen, zu Werthaltungen, zum moralischen Denken und Handeln, zum kritischen Denken etc. Viele an der Erziehung und Bildung beteiligte Personen würden sich mit Recht vehement dagegen wehren, wenn Erhebungen zu Moral und dergleichen durchgeführt würden. Und doch sind diese Dispositionen nicht weniger Erziehungs- und Bildungsziele wie die Kompetenzen. Ihre geringe Operationalisierbarkeit führt unterem dazu, dass in der Öffentlichkeit wenig Konsens über die anzustrebenden typischen Performanzen besteht; entsprechend sind sie als Ziele in den Lehrplänen zwar formuliert, aber zumindest in den Hauptfächern allenfalls in den Vorwörtern und auf sehr allgemeiner Ebene und nicht in jenen Kapiteln, wo es um die Konkretisierung des Unterrichts geht.

Folgt man dem Argument, nur das Messbare soll Ziel sein, darf sich Erziehung und Bildung nicht mit all den Bereichen befassen, die oben unter «typischer Performanz» zusammengefasst wurde. Dies lässt sich jedoch nicht rechtfertigen. Zum einen sind soziale Dispositionen, Werthaltungen, Einstellungen, moralisches Denken und Handeln, kritisches Denken untrennbar mit Unterricht verbunden: Was auch immer Lehrerinnen und Lehrer tun, sie beeinflussen ihre Schülerinnen und Schüler auch im Hinblick auf diese Dispositionen. Ethisch gesehen ist es da jedenfalls besser, in diesen Bereichen im erwünschten Sinne zu beeinflussen - wobei die Ziele natürlich zu reflektieren und im Sinne der «warranted assertibility» zu begründen sind -, als es dem Zufall zu überlassen, was die Schülerinnen und Schüler in dieser Hinsicht lernen, mit dem im gegenwärtigen Schulsystem zweifellos vorhandenen Risiko, dass sie genau das lernen, was niemand wünscht; genannt sei etwa, dass die Schülerinnen und Schüler lernen, dass sich Konflikte mit Gewalt «lösen» lassen.

Wenn in den Bildungsstandards aus Gründen der mangelnden Messbarkeit auf diese Ziele verzichtet wird und damit kommuniziert wird, sie seien irrelevant - was besonders beim oben erwähnten «teaching to the test» unvermeidlich ist erfolgt eine unverantwortliche Kürzung der Ziele. Das gleiche gilt, in geringerem Masse, für anspruchsvolle Kompetenzziele. Das Risiko besteht, dass nur Standards formuliert werden, die in der Taxonomie von Bloom, Englehart, Furst, Hill \& Krathwohl, (1956) als niedrig bezeichnet werden: Wissen, Verstehen, allenfalls Anwenden. Höhere Ziele wie Synthese, Analyse und Evaluation sind nur schwer zu messen und drohen deshalb ausser Acht gelassen zu werden. Die Entwicklung von Messinstrumenten für höhere Ziele ist ausgesprochen aufwendig (Shavelson \& Baxter, 1992).

Dies gilt in noch ausgeprägterem Masse für die assessment culture, die Herzog in Fussnote 5 anspricht (Segers, Dochy \& Cascallar, 2003) und die auf der Ebene der Schülerinnen und Schüler (nicht des Schulsystems) wünschbar wäre, 
ist noch anspruchsvoller und im schulischen Alltag nur beschränkt zu realisieren - und auf nationaler Ebene und bereichsübergreifend schon gar nicht, geht es doch letztlich darum, das assessment für die Regelung (im oben diskutierten Sinne) einzusetzen, letztlich im Hinblick auf eine Entsprechung zwischen dem, was die Schülerinnen und Schüler in der Schule tun, und dem, was von ihnen nach dem Schulabschluss erwartet wird, zu erhöhen; eine solche Konzeption ist für nationale Standards undenkbar.

\section{Zur technologischen Reform}

Herzog mahnt ein, bei Reformen nicht auf das kritische Denken zu verzichten. Dem ist vorbehaltlos zuzustimmen. Das kritische Denken obliegt jedoch nicht nur den Wissenschaftlern, sondern sollte auch den Politikern eine Selbstverständlichkeit sein. Dass diese dabei wissenschaftliche Erkenntnisse in ihre Überlegungen einbeziehen, ist nur zu begrüssen.

Auf die Frage nach der Technologisierbarkeit pädagogischer Prozesse (Kapitel 5.1) möchte ich nicht eingehen; die Frage ist viel zu komplex, als dass sie im Rahmen des hier verfügbaren Platzes angemessen abgehandelt werden könnte. Nur soviel: Jede Handlung - auch jede erzieherische Handlung - zielt darauf ab, etwas zu bewirken; wir haben Erwartungen darüber, mit welchen Mitteln wir welchen Zustand herbeiführen können. Manchmal sind wir erfolgreich, manchmal nicht. Wäre dem nicht so, müssten wir mangels Vorhersagbarkeit auf jede (erzieherische) Aktivität verzichten. Aber selbstverständlich muss das Argumentieren im Sinne der warranted assertibility der Komplexität, dem Gestalt- und dem Systemcharakter, etc., pädagogischer Prozesse Rechnung tragen. Hier ist nun Herzog wieder zuzustimmen, dass dies im Hinblick auf die Bildungsstandards wohl ungenügend, wenn überhaupt, der Fall ist.

Dass die gute Schule nicht nur am Output gemessen werden können (Abschnitt 5.2), ist sicher richtig, auch wenn Schule und mit ihr gutes Klima etc. nicht Selbstzweck sind, sondern letztlich im Dienste der Schülerinnen und Schüler und der Gesellschaft stehen sollen. Aber wenn wir schon die Outputs nicht messen können (vgl. oben), so können wir doch hoffen, dass ein gutes Klima besser dazu beiträgt, dass die Schülerinnen Dispositionen wie kritisches Denken, Verantwortungsbewusstsein etc. (vgl. oben, typische Performanz) entwickeln, als es ein schlechtes Klima tun würde.

Allerdings ist ein gutes Klima auch (aber nicht nur) von Bedeutung, weil Schülerinnen und Schüler nicht nur ein Recht auf die Zukunft haben, sondern auch in der Gegenwart leben und den berechtigten Anspruch haben, dass diese Gegenwart nicht belastend sei (Klafki, 1973, S. 53-91). Dieses "Moment des Zwecklosen und an sich Wertvollen» (Hervorhebung von Herzog) darf aber nicht überbewertet werden: Schule hat eine gesellschaftliche Bedeutung und ist nicht Selbstzweck, sonst müsste sie angesichts des Aufwandes, der für sie betrieben wird, aus ökonomischen Gründen abgeschafft werden; das aber will niemand. 


\section{Diskussion}

«Warranted assertibility» wurde oben als das zentrale Element von Wissenschaft bezeichnet. Politik machen bedeutet aber nicht, auf entsprechende Begründungen für Entscheidungen zu verzichten. Daher ist es nur folgerichtig, wenn sich Politiker dabei von Wissenschaftlern unterstützen lassen. Diese Kooperation kann aber weder bedeuten, dass die Politiker das Denken an die Wissenschaftler delegieren, noch umgekehrt dass die Wissenschaftler den Politikern das Denken überlassen dürfen. Vielmehr ist das kritische Denken gegenseitig einzumahnen, wobei die unterschiedlichen Argumentationskulturen gegenseitig anzuerkennen sind. In diesem Sinne ist die Diskussion um die Bildungsstandards, die von Herzog angeregt wurde, ein Lehrstück in Sachen Kooperation zwischen Politik und Wissenschaft. Die Kooperation ist wichtig und notwendig, aber die Umsetzung dieser Kooperation ist keineswegs trivial, sondern mit vielen Fallen gespickt. Einige der möglichen Probleme wurden angesprochen.

Allerdings muss die Diskussion über diese Kooperation ebenfalls den Prinzipien der warranted assertibility und der unterschiedlichen Argumentationskulturen genügen. Mit fragwürdigen Analogien - etwa zwischen der Maschinengewehrherstellung und Erziehung - und mit darauf aufbauenden Unterstellungen wird diesem Anspruch hingegen nicht entsprochen. Die Berücksichtigung unterschiedlicher Argumentationskulturen kann auch nicht bedeuten, dass Wissenschaftler so argumentieren, wie es unter Politikern häufig vorkommt: mittels Polemik und Diffamierungen. Das gute Klima ist nicht nur in der Schule (auch) an sich wertvoll, sondern dieser Anspruch gilt auch für wissenschaftliche Argumentation. Im Hinblick auf diese verschiedenen Punkte hätte ich mir im Artikel von Herzog bei inhaltlich gleicher Botschaft - über die man argumentieren kann - eine andere Diktion gewünscht. Polarisierende Aussagen wie von Herzog mögen Standpunkte deutlich machen, sie sind aber wenig konstruktiv. Letztlich geht es aber um die Schülerinnen und Schüler und darum, ihnen eine optimale Bildung zu ermöglichen; ich bezweifle, dass dieser Artikel trotz seiner unbezweifelten Berechtigung dazu beiträgt.

\section{Anmerkung}

1 Ist das auch ein Ladenhüter der Forschung, analog zu Ingenkamps (1970) klassischen Untersuchungen, die von Herzog so bezeichnet werden (Fussnote 5), darauf anspielend, dass diese Untersuchungen einige Jahrzehnte zurückliegen. Miller et al. (1960) liegt noch weiter zurück.

\section{Literatur}

Bloom, B. S., Englehart, M. B., Furst, E. J., Hill, W. H. \& Krathwohl, D. R. (1956). Taxonomy of educational objectives. The classification of educational goals. Handbook I: Cognitive domain. New York: Longmans Green.

Cronbach, L. J. (1970). Essentials of psychological testing (3d ed., p.35). New York: Harper \& Row. 
Gastager, A. \& Patry, J.-L. (2006). 'Allen Leuten recht getan ...'. Widersprüchliche Anforderungen an Evaluatorinnen und Evaluatoren. In W. Böttcher, H. G. Holtappels \& M. Brohm (Hrsg.), Evaluation im Bildungswesen. Eine Einführung in Grundlagen und Praxisbeispiele. Weinheim und München: Juventa.

Hanson, N. R. (1958). Patterns of discovery: An inquiry into the conceptual foundations of science. Cambridge, Mass.: Cambridge University Press.

House, E. R. (1993). Professional Evaluation: Social Impact and Political Consequences. Newbury Park, CA: Sage.

House, E. R. \& Howe, K. (2000). «Deliberative Democratic Evaluation.» In K. E. Ryan \& L. DiStefano (Eds.), Evaluation as a Democratic Process: Promoting Inclusion, Dialogue, and Deliberation (pp. 3-12). New Directions for Evaluation, no. 85. San Francisco, CA: JosseyBass.

Klafki, W. (1973). Das Pädagogische Verhältnis. In W. Klafki, G. Rückriem, W. Wolf, R. Freudenstein, H. K. Beckmann, K. C. Lingelbach, G. Iben, \& J. Diederich (Hrsg.), FunkKolleg Erziehungswissenschaft Band 1 (S. 53-91). Frankfurt: Fischer.

Mager, R. F. (1977). Lernziele und Unterricht. Weinheim: Beltz (völlig überarbeitete Neuauflage).

Miller, G. A., Galanter, E. H. \& Pribram, K. H. (1960). Plans and the Structure of Behavior. New York: Holt Rinehart \& Winston.

Mischel, W. (1968). Personality and Assessment. New York: Wiley.

Patry, J.-L. (1991). Transsituationale Konsistenz desVerhaltens und Handelns in der Erziehung. Bern: Lang.

Patry, J.-L. (1997). Eine Person - mehrere Werte. Überlegungen zum intrapersonalem Wertpluralismus. Pädagogische Rundschau, 51, 63-81.

Patry, J.-L. (2006). Die Werturteilsproblematik in der Erziehungswissenschaft. In G. Zecha (Hrsg), Werte in den Wissenschaften (S. 279-313).Tübingen: Mohr Siebeck.

Patry, J.-L. \& Patry, P. (2003). Werte und Normen in den Sozialwissenschaften. In A. Hiecke \& O.Neumaier (Hrsg.), Philosophie im Geiste Bolzanos, anlässlich des 222. Geburtstages von Bernard Bolzano, Edgar Morscher gewidmet (S. 199-222). Sankt Augustin: Academia.

Patry, J.-L., Harter, S., Höller, M., Höllhuber, R., Imani-Geyer, M., Uibner, M., Watschinger, M. \& Widmer, M. (2006). Feedback-Schlaufen: Von der Rückmeldung in der Unterrichtssituation bis zum Forschungsauftrag - eine Herausforderung für die Lehrerbildung. In C. Giordano \& J.-L. Patry (Hrsg.), Theorie und Praxis - Brüche und Brücken (S. 9-27). Münster: Lit.

Phillips, D. C. \& Burbules, N. C. (2000). Postpositivism and educational research (p. 31). New York: Rowman \& Littlefield.

Segers, M., Dochy, F. \& Cascallar, E. (Eds.). (2003). Optimising new modes in assessment: In search of qualities and standards. Dodrecht: Kluwer.

Shavelson, R. J. \& Baxter, G. P. (1992). Linking assessment with instruction. In F. Oser, A. Dick \& J.-L.Patry (Eds.), Effective and responsible teaching: The New Synthesis (pp. 80-90). San Francisco: Jossey-Bass,

Zecha, G. (1984). Für und wider die Wertfreiheit der Erziehungswissenschaft. München: Fink und Paderborn: Schöningh.

Schlagworte: Warranted assertibility, Theorie, Standards, Ziele, Normen, maximale und typische Performanz, Messbarkeit 